\title{
SOME SINGULAR, NONLINEAR, INTEGRAL EQUATIONS ARISING IN PHYSICAL PROBLEMS*
}

\author{
BY \\ I. LERCHE \\ University of South Carolina
}

\begin{abstract}
We show that if $Q$, the logarithmic decrement of plane wave intensity with position, is measured as a function of frequency, then the in-phase and quadrature components of the medium's characteristic response function (in the form of a dielectric function for electromagnetic waves, an elastic response function for acoustic waves, etc.) satisfy a nonlinear, singular, integral equation with the in-phase and quadrature components connected through a causal Hilbert transform pair.

Exact solutions of these inverse equations are extremely difficult to determine for a prescribed $Q$, but are of great value since decrement measurements are relatively easy to make compared to phase measurements.

For the electromagnetic and acoustic cases we show how to construct approximate solutions and we also show that the inverse equations for these two cases are very different in structure. We account for this structural difference in terms of the underlying physical manipulations performed on the system in order to extract a wave equation.

In view of the potential importance for yielding information on the behavior of the medium's response function from intensity measurements alone, we believe that this general class of inverse problem is deserving of wider attention than it seems to have been accorded to date.
\end{abstract}

1. Introduction. One of the more common ways of assessing the spatial scale of decay of a plane wave propagating in a homogeneous medium is simply illustrated using the vector electromagnetic equation

$$
\boldsymbol{\nabla} \times(\boldsymbol{\nabla} \times \mathbf{E})+c^{-2} \partial^{2} \mathbf{D} / \partial t^{2}=0 .
$$

With a constitutive relation between the displacement $\mathbf{D}$ and the electric field $\mathbf{E}$ in the usual form (see, e.g., Jackson [3])

$$
\mathbf{D}(\mathbf{x}, t)=\int_{-\infty}^{t} \varepsilon\left(t-t^{\prime}\right) \mathbf{E}\left(\mathbf{x}, t^{\prime}\right) d t^{\prime}
$$

\footnotetext{
*Received April 15, 1985.
} 
it follows that we can write the Fourier transform relations

$$
\mathbf{D}(\mathbf{x}, \omega)=\varepsilon(\omega) \mathbf{E}(\mathbf{x}, \omega)
$$

and

$$
\nabla \times(\nabla \times \mathbf{E})+\omega^{2} \varepsilon c^{-2} \mathbf{E}=0,
$$

where the real and imaginary parts of $\varepsilon(\omega)\left(\varepsilon=\varepsilon_{R}+i \varepsilon_{I}\right)$ are to be connected by the Hilbert transform pair

$$
\begin{aligned}
& \varepsilon_{I}(\omega)=\pi^{-1} P \int_{-\infty}^{\infty} \varepsilon_{R}\left(\omega^{\prime}\right)\left(\omega^{\prime}-\omega\right)^{-1} d \omega^{\prime} \\
& \varepsilon_{R}(\omega)=-\pi^{-1} P \int_{-\infty}^{\infty} \varepsilon_{I}\left(\omega^{\prime}\right)\left(\omega^{\prime}-\omega\right)^{-1} d \omega^{\prime},
\end{aligned}
$$

in order to satisfy causality [2-4], and where the Fourier transform is defined by $F(t)=\int_{-\infty}^{\infty} d \omega F(\omega) e^{-i \omega t}$. Then with the plane wave spatial variation $\exp (i k x)$, Eqs. (3) and (4) yield the dispersion relation

$$
c^{2} k^{2}=\omega^{2} \varepsilon .
$$

Split $k$ into its real and imaginary parts, $k=k_{R}+i k_{I}$, so that

$$
\begin{aligned}
c^{2}\left(k_{R}^{2}-k_{I}^{2}\right) & =\omega^{2} \varepsilon_{R}, \\
2 c^{2} k_{R} k_{I} & =\omega^{2} \varepsilon_{I} .
\end{aligned}
$$

Hence we have

$$
k_{R}=2^{-1 / 2} \omega c^{-1}\left[\varepsilon_{R}+\left(\varepsilon_{R}^{2}+\varepsilon_{I}^{2}\right)^{1 / 2}\right]^{1 / 2}
$$

and

$$
k_{I}=\frac{1}{2} \omega^{2} c^{-2} \varepsilon_{I} k_{R}^{-1} .
$$

Thus, given $\varepsilon_{R}(\omega)$ for all $\omega$ [or $\varepsilon_{I}(\omega)$ ], we can compute the real and imaginary parts of the wave number, representing phase variation and penetration depth of the wave since $\varepsilon_{I}$ (or $\varepsilon_{R}$ ) can be constructed from the appropriate Hilbert relation.

Acoustic waves satisfying Newton's equation

$$
\rho \frac{\partial^{2} \xi}{\partial t^{2}}=-\nabla p
$$

relating density $\rho$, displacement $\xi$, and pressure $p$, as well as the constitutive relation (see, e.g., Aki and Richards, [1, Vol. 1, Sec. 5])

$$
p(\mathbf{x}, t)=-\int_{-\infty}^{t} \kappa\left(t-t^{\prime}\right) \nabla \cdot \xi\left(\mathbf{x}, t^{\prime}\right) d t^{\prime}
$$

can be written in the Fourier form

$$
\rho \omega^{2} \xi=\nabla p
$$

and

$$
p=-\kappa(\omega) \nabla \cdot \xi
$$


so that the acoustic wave equation is

$$
\nabla \cdot\left(\rho^{-1} \nabla p\right)+\omega^{2} \kappa(\omega)^{-1} p=0 .
$$

Again, in order to satisfy causality we have

$$
\kappa=K_{R}+i K_{I}
$$

with $K_{R}$ and $K_{I}$ connected by the Hilbert transform pair

$$
\begin{aligned}
& K_{I}(\omega)=\pi^{-1} P \int_{-\infty}^{\infty} K_{R}\left(\omega^{\prime}\right)\left(\omega^{\prime}-\omega\right)^{-1} d \omega^{\prime}, \\
& K_{R}(\omega)=-\pi^{-1} P \int_{-\infty}^{\infty} K_{I}\left(\omega^{\prime}\right)\left(\omega^{\prime}-\omega\right)^{-1} d \omega^{\prime} .
\end{aligned}
$$

For a constant-density medium, plane wave solutions to the acoustic equation with $p \propto \exp (i k x)$ satisfy the dispersion relation

$$
k^{2}=\omega^{2} \rho / k=\omega^{2} \rho|K|^{-2}\left(K_{R}-i K_{I}\right),
$$

so that, splitting the wave number into its real and imaginary parts, $k=k_{R}+i k_{I}$, we have

$$
\begin{aligned}
k_{R}^{2}-k_{I}^{2} & =\omega^{2} \rho K_{R} /|K|^{2}, \\
2 k_{R} k_{I} & =-\omega^{2} \rho K_{I} /|K|^{2},
\end{aligned}
$$

with solution

$$
\begin{aligned}
k_{R} & =2^{-1 / 2} \omega \rho^{1 / 2}|K|^{-1}\left[K_{R}+\left(K_{R}^{2}+K_{I}^{2}\right)^{1 / 2}\right]^{1 / 2}, \\
k_{I} & =-\frac{1}{2} \omega^{2} \rho|K|^{-2} K_{I} k_{R}^{-1} .
\end{aligned}
$$

It would seem then that the differences in behavior between acoustic and electromagnetic waves are matters of precise functional forms for $\varepsilon_{R}$ (vs. $K_{R}$ ). Given $\varepsilon_{R}(\omega)$ (or $K_{R}$ ), the calculation of $\varepsilon_{I}(\omega)\left[K_{I}(\omega)\right]$ proceeds through using the Hilbert transform relations; $k_{R}$ and $k_{I}$ are then computed, and the behavior of the phase and effective spatial attenuation of the wave with angular frequency $\omega$ can be simply determined.

To this point the differences in fundamental behavior, either physically or mathematically, between the two wave types seem trite.

In fact, however, a very subtle difference between manipulations on the two wave equations is present, which is most easily brought to light through an inverse problem.

Pragmatically, the spatial attenuation of seismic waves in the subsurface is often measured by recording the wave intensity with depth $\left(\alpha \exp \left(-2 k_{I} x\right)\right)$ as a function of frequency. Then measurements at two depths allow us to determine $k_{I}(\omega)$.

In conventional terms the frequency-dependent quality factor $Q(\omega)$ is normally presented as a function of frequency with

$$
2 k_{I}(\omega)=\omega /(V Q(\omega))
$$

where $V$ is a constant sound speed. The inverse problem is: given $Q(\omega)$ for all $\omega$, is it possible to determine the real and imaginary parts, $K_{R}(\omega)$ and $K_{I}(\omega)$, of the elastic "constant" and so to learn something about intrinsic rock properties in the subsurface? 
More generally: given $Q(\omega)$ for either acoustic plane waves or electromagnetic waves, can we "invert" the relations connecting $k_{I}$ and $k_{R}$ to determine $\varepsilon_{R}$ and $\varepsilon_{I}\left(K_{R}\right.$ and $\left.K_{I}\right)$ when the causal relations between $\varepsilon_{R}$ and $\varepsilon_{I}\left(K_{R}\right.$ and $\left.K_{I}\right)$, expressed by the Hilbert transform pairs, are maintained?

We address this problem here. As we shall see, the relevant inversions require that we have the capability of solving nonlinear singular integral equations which are extremely different in form for the electromagnetic and acoustic cases. As a consequence we shall consider each case separately.

\section{Inversion in terms of constitutive equations}

a. The electromagnetic equation. Suppose we are given the spatial decay of plane wave intensity in a medium, at all frequencies, in the form

$$
k_{I}=\frac{1}{2} \omega c^{-1} Q(\omega)^{-1}>0
$$

so that $Q(\omega)$ is known and positive for all $\omega$. Then from Eq. (6b) we have

$$
k_{R}=\omega c^{-1} \varepsilon_{I} Q(\omega) .
$$

Substitute (19) and (20) into (6a), obtaining

$$
\varepsilon_{l}^{2} Q(\omega)^{4}-\varepsilon_{R} Q(\omega)^{2}-\frac{1}{4}=0 .
$$

But in order to satisfy causality, $\varepsilon_{I}(\omega)$ and $\varepsilon_{R}(\omega)$ are to be connected through the Hilbert transform relations (5a) and (5b). Hence we can rewrite Eq. (21) in the form of a nonlinear singular integral equation

$$
\varepsilon_{I}(\omega)^{2} Q(\omega)^{4}+\pi^{-1} Q(\omega)^{2} P \int_{-\infty}^{\infty} \varepsilon_{I}\left(\omega^{\prime}\right)\left(\omega^{\prime}-\omega\right)^{-1} d \omega^{\prime}-\frac{1}{4}=0
$$

with $Q(\omega)$ known.

We require solutions to Eq. (22) for $\varepsilon_{I}(\omega)$ in terms of $Q(\omega)$. The corresponding $\varepsilon_{R}(\omega)$ is then trivially given as a quadrature through the Hilbert relation (5b).

Let us leave this equation to one side for the moment and develop the corresponding integral equation for acoustic waves.

b. The acoustic equation. Suppose we are given the spatial decay of acoustic intensity in a medium of constant density, at all frequencies, in the form

$$
k_{I}=\frac{1}{2} \omega /(V Q(\omega))>0
$$

so that $Q(\omega)$ is known, and positive, for all $\omega$. In addition, we take the acoustic velocity to be constant, independent of frequency. We can use the form (23) in Eq. (16b) to obtain

$$
k_{R}=-\omega V Q \rho K_{I}|K|^{-2},
$$

and we can substitute (23) and (24) into Eq. (16a) to obtain

$$
|K|^{4}+4 \rho K_{R}(V Q)^{2}|K|^{2}-4 \rho^{2}(V Q)^{4} K_{I}^{2}=0,
$$

of which the formal solution can be written

$$
K_{R}^{2}+K_{I}^{2}=4 \rho(V Q)^{2}\left[|K|-K_{R}\right]>0
$$


or, using the Hilbert relation (14a), we can write (26) in the form of a nonlinear integral equation

$$
\begin{aligned}
& K_{R}(\omega)^{2}+\pi^{-2}\left[P \int_{-\infty}^{\infty} K_{R}\left(\omega^{\prime}\right)\left(\omega^{\prime}-\omega\right)^{-1} d \omega^{\prime}\right]^{2}+4 \rho(V Q(\omega))^{2} K_{R}(\omega) \\
& \quad-4 \rho(V Q(\omega))^{2}\left[K_{R}(\omega)^{2}+\pi^{-2}\left[P \int_{-\infty}^{\infty} K_{R}\left(\omega^{\prime}\right)\left(\omega^{\prime}-\omega\right)^{-1} d \omega^{\prime}\right]^{2}\right]^{1 / 2}=0 .
\end{aligned}
$$

The structure of Eq. (27) is very different from the structure of Eq. (22), so the similarities between the electromagnetic equation and the acoustic equation, so obvious in Sec. 1, are now minimized while the differences are maximized. The physical reason for the very different structural behaviors of Eqs. (22) and (27) can be traced back to the original equations and constitutive relations. Crudely speaking, in the electromagnetic case we specify a relation between the displacement vector ("pondermotive force") in terms of the fundamental electric field. We then eliminate the pondermotive field, $\mathbf{D}$, from the picture in favor of $\mathbf{E}$. In the acoustic case we again specify a relation between the driving force (pressure) and the intrinsic displacement field-but then we eliminate the fundamental displacement field from the picture in favor of the pressure $p$.

An alternative way to view this difference in behavior is to note that in the acoustic case we specify a relation between the scalar pressure field $p$ and the vector displacement field $\boldsymbol{\xi}$, while in the electromagnetic case we specify a relation between the vector field $\mathbf{D}$ and the vector field $\mathbf{E}$. Hence in the acoustic case, while it is possible to write a wave equation for the vector displacement $\boldsymbol{\xi}$ as opposed to the scalar pressure $p$, as we have done, nevertheless one would have to split $\boldsymbol{\xi}$ into longitudinal and transverse components to obtain a solution, with the longitudinal component being essentially the pressure field (since $p \propto \nabla \cdot \xi$, so the transverse component will not contribute). In the electromagnetic case we deal directly with a vector wave equation with $\nabla \cdot \mathbf{D}=0$, so we deal only with transverse waves and not longitudinal waves. So from the "pondermotive" viewpoint, the constitutive relation viewpoint, and the viewpoint of the basic nature of the waves, we might anticipate a difference in behavior.

The question before us from a mathematical and physical point of view is whether we can solve the inverse Eqs. (22) and (27) given the behavior of the respective $Q(\omega)$.

3. The linear approximation. To date, we have found Eqs. (22) and (27) too difficult to solve in complete generality for arbitrary, positive $Q(\omega)$, although particular solutions can be obtained when $Q(\omega) \propto \omega^{\lambda}$ for restricted ranges of $\lambda$. However, when $Q(\omega)$ is considered to be a slowly varying function of $\omega$ (in a manner to be defined a posteriori) it is possible to make some progress with Eqs. (22) and (27). We consider each in turn.

a. The electromagnetic equation (22). Let

$$
Q(\omega)=Q_{0}+\delta Q(\omega),
$$

and let

$$
\varepsilon_{I}=\varepsilon_{0}+\delta \varepsilon(\omega)
$$


where $\varepsilon_{0}$ and $Q_{0}$ are independent of $\omega$. Then from (22) we obtain

$$
\left(\varepsilon_{0}^{2} Q_{0}^{2}-\frac{1}{4}\right)+Q_{0}^{2}\left\{2 Q_{0}^{2} \varepsilon_{0} \delta \varepsilon+4 \varepsilon_{0} Q_{0}^{3} \delta Q+\pi^{-1} P \int_{-\infty}^{\infty} \delta \varepsilon\left(\omega^{\prime}\right)\left(\omega^{\prime}-\omega\right)^{-1} d \omega^{\prime}\right\}=0
$$

to order $\delta \varepsilon$ and $\delta Q$.

The frequency-independent part of Eq. (30) provides

$$
\varepsilon_{0}=\frac{1}{2} Q_{0}^{-2},
$$

while the frequency-dependent part can be written

$$
\delta \varepsilon(\omega)+\pi^{-1} P \int_{-\infty}^{\infty} \delta \varepsilon\left(\omega^{\prime}\right)\left(\omega^{\prime}-\omega\right)^{-1} d \omega^{\prime}=-2 \delta Q(\omega) / Q_{0} .
$$

Equation (32) has the solution [6]

$$
\delta \varepsilon(\omega)=-\delta Q(\omega) / Q_{0}+\pi^{-1} P \int_{-\infty}^{\infty}\left(\delta Q\left(\omega^{\prime}\right) / Q_{0}\right)\left(\omega^{\prime}-\omega\right)^{-2} d \omega^{\prime}
$$

so that

$$
\varepsilon_{I}=\frac{1}{2} Q_{0}^{-2}+\delta \varepsilon(\omega)
$$

and

$$
\varepsilon_{R}=\delta Q(\omega) / Q_{0}+\pi^{-1} P \int_{-\infty}^{\infty}\left(\delta Q\left(\omega^{\prime}\right) / Q_{0}\right)\left(\omega^{\prime}-\omega\right)^{-1} d \omega^{\prime} .
$$

Consider now the acoustic wave behavior.

$d$. The acoustic equation (27). Here we follow a different approach than in the electromagnetic case since there is no term in Eq. (27) independent of $k_{R}$ or $k_{I}$, whereas in the electromagnetic case [Eq. (22)] the last term on the left-hand side was constant.

Rewrite Eq. (27) as

$$
|K|^{2}-\alpha^{2}|K|+\alpha^{2} K_{R}=0,
$$

where $\alpha=2 V Q(\omega)>0$. Then

$$
|K|=\frac{1}{2} \alpha^{2}\left[1 \pm\left(1-4 K_{R} \alpha^{-2}\right)^{1 / 2}\right] .
$$

Since $K$ is real, it follows from (37) that an overriding requirement on any solution is

$$
4 K_{R}<\alpha^{2} .
$$

Since, further, $|K|>0$ it follows that if we choose the lower sign in Eq. (37) then we have the further restriction

$$
0<K_{R}<\alpha^{2} / 4 \text {. }
$$

With

$$
K_{R}=-\pi^{-1} H K_{I}, \quad K_{I}=\pi^{-1} H K_{R},
$$


where $H$ is the Hilbert transform operation

$$
H f(\omega) \equiv P \int_{-\infty}^{\infty} f\left(\omega^{\prime}\right)\left(\omega^{\prime}-\omega\right)^{-1} d \omega^{\prime},
$$

an approximate solution to Eq. (36) can be found as follows.

Note that if we had been dealing with the constant $Q$ case (constant $\alpha$ ) then a solution to Eq. (36) would have been

$$
K_{I}=\alpha, \quad K_{R}=0 .
$$

The Hilbert relations (40) are then satisfied modulo a constant because the Hilbert transform of a constant is zero. Also $[2,6]$

$$
H(H f)=-\pi^{2} f, \quad \bmod (\text { constant }) .
$$

Suppose then that $Q$ varies slowly. Assume $\left|K_{R}\right| \ll\left|K_{I}\right|$ so that we can write Eq. (36) in the approximate form

$$
K_{I}-\alpha^{2} \simeq-\alpha^{2} K_{R} K_{I}^{-1}+\frac{1}{2} \alpha^{2}\left(K_{R} / K_{I}\right)^{2}-K_{R}^{2} K_{I}^{-1}
$$

The lowest-order behavior to Eq. (44) yields the approximate solution

$$
K_{l}^{(0)} \simeq \alpha^{2}(\omega) \text {. }
$$

Use of the Hilbert transform relations (40) then yields the equivalent level of solution

$$
K_{R}^{(0)}=K_{0}-\pi^{-1} H \alpha^{2}(\omega),
$$

where $K_{0}$ is a, so far, arbitrary constant.

Use of (45a) and (45b) on the right-hand side of (44) yields the next order of approximation

$$
K_{I}^{(1)}=\alpha^{2}-K_{R}^{(0)}+\left(\frac{1}{2}-\alpha^{-2}\right)\left(K_{R}^{(0)}\right)^{2},
$$

while the Hilbert transform relations (40) then provide

$$
K_{R}^{(1)}=K_{0}-\pi^{-1} H \alpha^{2}+\alpha^{2}-\pi^{-1} H\left\{\left[\frac{1}{2}-\alpha^{-2}\right]\left[K_{0}-\pi^{-1} H \alpha^{2}\right]^{2}\right\} \text {. }
$$

Since $\alpha$ is considered to be a slowly varying function, and since our initial premise was that $\left|K_{R}\right| \ll\left|K_{I}\right|$, it follows that $K_{0}$ must be chosen to enforce $\left|K_{R}\right| \ll\left|K_{I}\right|$. For $\alpha$ slowly varying, one such choice of $K_{0}$ is obtained by minimizing some appropriate weighted functional of $\left|K_{R}^{(1)} / K_{I}^{(1)}\right|$, integrated over all $\omega$, with respect to $K_{0}$. Other choices are also available (e.g., let $K_{0}$ be such that $\left|K_{R}^{(1)} / K_{I}^{(1)}\right| \rightarrow 0$ as $\omega \rightarrow \infty$, etc.)

The point is that it is always possible to find a value of $K_{0}$ such that the original premise is valid.

A more exact method of solving the singular nonlinear integral Eq. (36) than we have been able to find would presumably also provide a better method of determining $K_{0}$.

4. Discussion and conclusion. The similarity between transverse electromagnetic plane waves and scalar acoustic plane waves propagating in a medium, so often noted in the past, is strongly depressed when we consider the inverse problem of measuring the spatial 
decay of intensity at all frequencies and attempt to determine the complex dielectric function or complex elastic function. The differences between the two types of waves are then emphasized: the electromagnetic wave equation is for the basic field $\mathbf{E}$ rather than the pondermotive field $\mathbf{D}$; in the acoustic case we deal with a wave equation for the equivalent pondermotive field $p$ rather than the basic displacement $\xi$. It is this difference which, when coupled to the analytically causal Hilbert transform relations for the constitutive relations between $\mathbf{D}$ and $\mathbf{E}$ ( $p$ and $\boldsymbol{\xi}$ ), amplifies the fundamental difference in the inverse problems.

From a mathematical viewpoint the class of inverse problems uncovered, and exemplified by the acoustic and electromagnetic cases, leads to singular, nonlinear integral equations which are incredibly rich in structure and behavior [they are also very difficult to solve for prescribed $Q(\omega)$ !].

From a pragmatic point of view it is of some importance to deal with the inverse problem. The point here is that it is often very easy to measure the spatial decay of intensity of a wave with frequency and to leave its phase determination out of the picture entirely. If the inverse equations are invoked, and solutions obtained, we then learn a great deal about the behavior of the material parameters of the medium from relatively easily made measurements. The alternative procedure is to measure both the spatial attenuation and the phase of the wave with respect to frequency. Unfortunately, the so-called "phase unwrapping" problem is extremely sensitive to very precise and accurate phase measurements being made (see, e.g., Tribolet [5] and copious references therein).

To date, we have been able to supply only approximate solutions to the electromagnetic and acoustic inverse equations and, even then, only under the linear approximation framework given in the paper. We know very little about uniqueness of solutions of the inverse equations, and we know even less about constructing exact solutions to the singular, nonlinear integral equations.

It would seem that this class of inverse equations should enjoy a more detailed investigation than it has been given to date both from the challenge of a mathematical problem per se and because of the use to which solutions can be put in applied physics problems.

\section{REFERENCES}

[1] K. Aki and P. G. Richards, Quantitative seismology, theory and methods, Freeman, San Francisco (1980)

[2] R. Courant and D. Hilbert, Methods of mathematical physics, Interscience, New York (1953)

[3] J. D. Jackson, Classical electrodynamics, Wiley, New York (1962)

[4] P. M. Morse and H. Feshbach, Methods of theoretical physics, McGraw-Hill, New York (1953)

[5] J. M. Tribolet, Seismic applications of homomorphic signal processing, Prentice-Hall, Englewood Cliffs, N. J. (1979)

[6] F. G. Tricomi, Integral equations, Interscience, New York (1957) 